\title{
Bipartite Consensus of Linear Discrete-Time Multiagent Systems with Exogenous Disturbances under Competitive Networks
}

\author{
Yi Yuan, ${ }^{1}$ Shamrie Sainin Mohd ${ }^{1},{ }^{1}$ and Yanhui $\mathrm{Zhu}^{2}$ \\ ${ }^{1}$ Faculty of Computing and Informatics, Universiti Malaysia Sabah, 88400, Kota Kinabalu Sabah, Malaysia \\ ${ }^{2}$ School of Computer Science, Hunan University of Technology, Zhuzhou, 412008, China \\ Correspondence should be addressed to Shamrie Sainin Mohd; shamrie@ums.edu.my
}

Received 30 March 2021; Accepted 15 May 2021; Published 26 May 2021

Academic Editor: Hou-Sheng Su

Copyright (C) 2021 Yi Yuan et al. This is an open access article distributed under the Creative Commons Attribution License, which permits unrestricted use, distribution, and reproduction in any medium, provided the original work is properly cited.

This paper investigates the bipartite consensus of linear discrete-time multiagent systems (MASs) with exogenous disturbances. A discrete-time disturbance-observer- (DTDO-) based technology is involved for attenuating the exogenous disturbances. And both the state feedback and observer-based output feedback bipartite consensus protocols are proposed by using the DTDO method. It turned out that bipartite consensus can be realized under the given protocols if the topology is connected and structurally balanced. Finally, numerical simulations are presented to illustrate the theoretical findings.

\section{Introduction}

In the past two decades, the coordination of MASs has attracted much attention for its wide applications [1-4]. In coordination issues, consensus plays a very important role, which means that the final states of all agents can asymptotically reach a common value. Many works about consensus have been reported in the past few years, including consensus of MASs with different dynamics [5-10] and consensus of MASs via different control methods [11-16].

Many existing works mainly focus on the consensus of cooperative networks. However, in many real networks, there exist competitive relations between the nodes. Bipartite consensus was firstly investigated for MASs with antagonistic links [17], in which the nodes were divided into two parts, one will asymptotically track the leader and the other part will asymptotically converge to the reverse state of the leader. The topology of the discussed network was assumed as structurally balanced. And gauge transformation was used for solving the problems of stability analysis. Then, many efforts were devoted to bipartite consensus of MASs with antagonistic links or competitive topologies. Bipartite consensus was investigated for MASs with time-varying delay [18]. Bipartite edge consensus was studied for MASs with edge dynamics under the corresponding line graph spanned by the nodal graph [19]. Qin et al. [20] investigated global bipartite consensus of MASs with input saturation, and $\mathrm{Hu}$ et al. [21] solved bipartite consensus problems of MASs with communication noise. The event-triggered bipartite consensus was investigated for first-order MASs [22]. Under different topologies, event-triggered adaptive bipartite output consensus of heterogenous linear MASs was studied [23]. Considering the rate of convergency, finite-time bipartite consensus was investigated [24].

However, the above papers mainly investigated bipartite consensus of continuous-time MASs without disturbances. Disturbance often exists and is the main resource of poor performance of the controlled systems. In real networks, the subsystem in the network may be sufferred by exogenous disturbances. Therefore, research studies about multiagent systems with exogenous disturbances are very important and significant. By using a novel backstepping method, robust global coordination was investigated for MASs with input saturation and disturbances [25]. In [26], the disturbance- 
observer- (DO-) based control method was proposed for stabilizing nonlinear systems with exogenous disturbances. Using the continuous DO method proposed in [26], Yang et al. [27] solved the consensus problems of second-order MASs with exogenous disturbances. Containment control of continuous-time MASs with exogenous disturbances was investigated in virtue of the DO technique [28]. Intermittent consensus of MASs with exogenous disturbances was investigated by using both state feedback control and output feedback control protocols [29]. And the DO-based method is used on many other control plants [30, 31].

The existing relative works mainly focus on the consensus of MASs with continuous dynamics and cooperative topology. But there exist few results about bipartite consensus for discrete-time MASs with competitive topology and exogenous disturbances. Motivated by the above literatures, this paper investigates the bipartite consensus of discrete-time linear MASs with exogenous disturbances. The main contributions are as follows. (i) Discrete-time MASs are discussed in this paper, which are more challenging because the analysis of stability is more complex than the continuous-time MASs. (ii) Competitive network is considered in this paper, which can be used to describe more real network. And many results about the bipartite consensus of competitive networks can be applied into the consensus of cooperative networks. (iii) The DTDO-based method is used for attenuating the exogenous disturbances. Both the state feedback and output feedback control protocols are proposed in this paper.

The rest of the paper is organized as follows. Section 2 states the model considered in the paper and gives some basic lemmas and assumptions. In Section 3, discrete-time DO-based state feedback containment protocol is proposed. In Section 4, discrete-time DO-based output feedback containment protocol is given. Numerical examples are included to demonstrate the proposed protocol in Section 5. Finally, Section 6 gives a conclusion for this paper.

\section{Preliminaries and Model Description}

A network can be described by a graph $\mathscr{G}=(\mathscr{V}, \mathscr{E}, \mathscr{A})$, which includes a set of nodes $\mathscr{V}=\{1,2, \ldots, N\}$, a set of edges $\mathscr{E} \subseteq \mathscr{V} \times \mathscr{V}$, and an adjacent matrix $\mathscr{A}=\left[a_{i j}\right]$. For an undirected graph, $a_{i j}=a_{j i}$, for $i, j=1,2, \ldots, N$. $a_{i j} \neq 0 \Leftrightarrow(j, i) \in \mathscr{E} . \mathcal{N}_{i}=j \mid(j, i) \cdot \mathscr{E}$ is a neighbor set of $i$ th node. A path between node $v_{i_{1}}$ and node $v_{i_{n}}$ is formed by an array of edges $\left(v_{i_{1}}, v_{i_{2}}\right),\left(v_{i_{2}}, v_{i_{3}}\right), \ldots,\left(v_{i_{n-1}}, v_{i_{n}}\right)$. An undirected graph is connected if there exists a path between any two nodes. $L=\left[l_{i j}\right]_{N \times N}$ is the Laplacian matrix, where $l_{i i}=\sum_{j=1, j \neq i}^{N}\left|a_{i j}\right|, l_{i j}=-a_{i j}$.

The dynamics of the $i$ th follower are described as

$$
\begin{aligned}
x_{i}(k+1) & =A x_{i}(k)+B\left(u_{i}(k)+\gamma_{i}(k)\right), \\
y_{i}(k) & =C x_{i}(k), \\
i & =1,2, \ldots, N .
\end{aligned}
$$

And the dynamics of the leader are described as

$$
\begin{aligned}
x_{0}(k+1) & =A x_{0}(k), \\
y_{0}(k) & =C x_{0}(k),
\end{aligned}
$$

where $x_{i}(k) \in R^{n}, u_{i}(k) \in R^{p}, \gamma_{i}(k) \in R^{p}$, and $y_{i}(k) \in R^{m}$ denote the state, control input, exogenous disturbance, and output of the $i$ th follower, respectively, $x_{0}(k) \in R^{n}$ and $y_{0}(k) \in R^{m}$ denote the state and output of the leader, and $A \in R^{n \times n}, B \in R^{n \times p}$, and $C \in R^{m \times n}$ are constant matrices. It is assumed that the disturbance $\gamma_{i}(k)$ is generated by the following exogenous system:

$$
\begin{aligned}
\omega_{i}(k+1) & =S \omega_{i}(k), \\
\gamma_{i}(k) & =F \omega_{i}(k),
\end{aligned}
$$

where $\omega_{i} \in R^{l}$ is the state of the exogenous system and $S \in R^{l \times l}$ and $F \in R^{p \times l}$ are constant matrices.

The following assumptions and lemmas are necessary for the main results of this paper.

Definition 1 (see [24]). A signed graph $\mathscr{G}$ is said to be structurally balanced if the following hold:

(1) It admits a bipartition of nodes as $\mathscr{V}_{1}$ and $\mathscr{V}_{2}$, where $\mathscr{V}_{1} \cap \mathscr{V}_{2}=\varnothing, \mathscr{V}_{1} \cup \mathscr{V}_{2}=\mathscr{V}$

(2) The elements of $\mathscr{V}_{q}, q \in\{1,2\}$ have the following relation:

$$
a_{i j} \geq 0, \forall i, j \in \mathscr{V}_{q} ; a_{i j} \leq 0, \forall i \in \mathscr{V}_{q}, j \in \mathscr{V}_{r}, q \neq r .
$$

Otherwise, the graph $\mathscr{G}$ is structurally unbalanced.

Definition 2. The leader-following bipartite consensus of system (1) with leader (2) is said to be achieved, if there exists a protocol $u_{i}(k)$ such that

$$
\lim _{t \longrightarrow \infty}\left\|x_{i}(k)-d_{i} x_{0}(k)\right\|=0, \quad i=1,2, \ldots, N,
$$

for any initial condition $x_{l}(1), l=0,1, \ldots, N$, where $d_{i}=1$, if $i \in \mathscr{V}_{1} ; d_{i}=-1$, if $i \in \mathscr{V}_{2}$.

Remark 1. According to gauge transformation [17], denoting $D=\operatorname{diag}\left\{d_{1}, d_{2}, \ldots, d_{N}\right\}$, one has that $D L D$ is semipositive defined under the assumption that $G$ is connected.

Assumption 1. Suppose the undirected signed graph $\mathscr{G}$ is connected and structurally balanced.

Assumption 2. The matrix pair $(A, B)$ is stabilizable.

Assumption 3. The matrix pair $(A, C)$ is detectable.

Lemma 1 (see [32]). For any matrix $\Lambda=\operatorname{diag}(h, 0, \ldots, 0)$ with $h>0$, all eigenvalues of the matrix $D \widetilde{L} D=D L D+\Lambda$ are positive if Assumption 1 holds, where $\widetilde{L}=L+\Lambda$.

Lemma 2 (see [33]). Under Assumption 2, there exists a unique positive definite matrix $P$, satisfying the algebraic Riccati equation 


$$
A^{T} P A-P-A^{T} P B\left(B^{T} P B+I\right)^{-1} B^{T} P A+I=0 .
$$

Lemma 3 (see [34]). Suppose that $A, B, C$ are matrices with appropriate dimensions; then, the following inequalities are equivalent:
(1) $\left(\begin{array}{cc}A & B \\ B^{T} & C\end{array}\right)<0$.
(2) $A<0$ and $C-B^{T} A^{-1} B<0$.
(3) $C<0$ and $A-B C^{-1} B^{T}<0$.

\section{DO-Based State Feedback Bipartite Consensus}

In this section, based on the DTDO method, bipartite consensus of MAS with disturbances is solved by using relative state information.

A disturbance observer is designed as follows:

$$
\begin{aligned}
v_{i}(k+1) & =(S+H B F)\left(v_{i}(k)-H x_{i}(k)\right)+H\left(A x_{i}(k)+B u_{i}(k)\right), \\
\widehat{\omega}_{i}(k) & =v_{i}(k)-H x_{i}(k), \\
\widehat{\gamma}_{i}(k) & =F \widehat{\omega}_{i}(k),
\end{aligned}
$$

where $v_{i}(k) \in R^{l \times l}$ is the internal state variable of the observer, $\widehat{\omega}_{i}(k) \in R^{l}$ and $\hat{\gamma}_{i}(k) \in R^{p}$ are the estimated values of $\omega_{i}(k)$ and $\gamma_{i}(k)$, respectively, and $H \in R^{l \times n}$ is the gain matrix of the observer.

Remark 2. The agents in the network cannot get the information of the disturbances, which leads to that the agents have to estimate the value of the exogenous disturbances. A discrete disturbance observer (7) is proposed for estimating the disturbances.

According to (1) and (7), one has

$$
\begin{aligned}
\widehat{\omega}_{i}(k+1)= & v_{i}(k+1)-H x_{i}(k+1) \\
= & (S+H B F)\left(v_{i}(k)-H x_{i}(k)\right)+H\left(A x_{i}(k)\right) \\
& \left(+B u_{i}(k)\right)-H\left(A x_{i}(k)+B\left(u_{i}(k)\right)+\gamma_{i}(k)\right) \\
= & (S+H B F) \widehat{\omega}_{i}(k)-H B F \omega_{i}(k) .
\end{aligned}
$$

Then, denoting the state error of exogenous system as $e_{i}(k)$, one has

$$
\begin{aligned}
e_{i}(k+1) & =\omega_{i}(k+1)-\widehat{\omega}_{i}(k+1) \\
& =S \omega_{i}(k)-(S+H B F) \widehat{\omega}_{i}(k)+H B F \omega_{i}(k) \\
& =(S+H B F)\left(\omega_{i}(k)-\widehat{\omega}_{i}(k)\right) \\
& =(S+H B F) e_{i}(k) .
\end{aligned}
$$

Consider the following distributed bipartite consensus protocol for discrete-time MAS (1):

$$
u_{i}(k)=K\left[\sum_{j \in N_{i}}\left|a_{i j}\right|\left(x_{i}(k)-\operatorname{sgn}\left(a_{i j}\right) x_{j}(k)\right)+h_{i}\left(x_{i}(k)-d_{i} x_{0}(k)\right)\right]-\hat{\gamma}_{i}(k),
$$

where $K$ is the gain matrix to be determined.

Substituting (10) into (1), one has that by (7) and (9),

$$
x_{i}(k+1)=A x_{i}(k)+B F e_{i}(k)+B K\left[\sum_{j \in N_{i}}\left|a_{i j}\right|\left(x_{i}(k)\left(-\operatorname{sgn}\left(a_{i j}\right) x_{j}(k)\right)+h_{i}\left(x_{i}(k)-d_{i} x_{0}(k)\right)\right)\right] .
$$

Theorem 1. Suppose Assumptions 1 and 2 hold. The bipartite consensus of MAS (1) with leader (2) will be achieved by error system (9) with disturbance observer (7) under bipartite consensus protocol (10) if

(i) Suppose there exists at least one follower pinned by the leader.

(ii) $S+H B F$ is Schur stable. (iii) $K=-\left(1 / \lambda_{1}(D \tilde{L} D)\right)\left(B^{T} P B+I\right)^{-1} B^{T} P A$, where $P>0$ is the unique solution of algebraic Riccati equation (6), $\lambda_{1}(D \widetilde{L} D)$ is the minimum eigenvalue of $D \widetilde{L} D$,

$D \widetilde{L} D=D L D+\Lambda, \Lambda=\operatorname{diag}\left(h_{1}, h_{2}, \ldots, h_{N}\right)$.

and

Proof. Let $\quad \tilde{x}_{i}(k)=x_{i}(k)-d_{i} x_{0}(k)$. Because $d_{i} a_{i j} d_{j}=a_{i j} \operatorname{sgn}\left(a_{i j}\right)=\left|a_{i j}\right|$, one has

$$
\begin{aligned}
\tilde{x}_{i}(k+1) & =x_{i}(k+1)-d_{i} x_{0}(k+1) \\
& =A \widetilde{x}_{i}(k)+B F e_{i}(k)+B K\left[\sum_{j \in N_{i}}\left|a_{i j}\right|\left(\tilde{x}_{i}(k)-\operatorname{sgn}\left(a_{i j}\right) \tilde{x}_{j}(k)\right)+h_{i} \widetilde{x}_{i}(k)\right], \\
e_{i}(k+1) & =(S+H B F) e_{i}(k) .
\end{aligned}
$$


Denote $\quad \tilde{x}(k)=\left(\tilde{x}_{1}^{T}(k), \tilde{x}_{2}^{T} \quad(k), \ldots, \tilde{x}_{N}^{T}(k)\right)^{T}, e(k)=$ $\left(e_{1}^{T}(k), e_{2}^{T}(k), \ldots, e_{N}^{T}(k)\right)^{T}$. Then, (12) can be rewritten as follows:

$$
\begin{aligned}
& \tilde{x}(k+1)=\left(I_{N} \otimes A+\widetilde{L} \otimes B K\right) \widetilde{x}(k)+\left(I_{N} \otimes B F\right) e(k), \\
& e(k+1)=\left(I_{N} \otimes(S+H B F)\right) e(k) .
\end{aligned}
$$

Consider the following Lyapunov function candidate

$$
\begin{aligned}
V(k) & =\eta^{T}(k)\left(\begin{array}{cc}
I_{N} \otimes P & 0 \\
0 & \alpha I_{N} \otimes Q_{1}
\end{array}\right) \eta(k) \\
& =\tilde{x}^{T}(k)\left(I_{N} \otimes P\right) \tilde{x}(k)+\alpha e^{T}(k)\left(I_{N} \otimes Q_{1}\right) e(k),
\end{aligned}
$$

where $\eta(k)=\left(\tilde{x}^{T}(k), e^{T}(k)\right)^{T}, Q_{1}>0$ is a matrix designed later, and $\alpha$ is a large enough positive constant.

Then,
Let $\bar{x}(k)=\left(D \otimes I_{n}\right) \tilde{x}(k)$ and $\bar{\eta}(k)=\left(\bar{x}^{T}(k), e^{T}(k)\right)^{T}$.

$$
\begin{aligned}
V(k+1)-V(k)= & \bar{x}^{T}(k)\left(I_{N} \otimes A^{T} P A+2 D \widetilde{L} D \otimes A^{T} P B K\right) \\
& +\left((D L D)^{2} \otimes K^{T} B^{T} P B K-I_{N} \otimes P\right) \bar{x}(k) \\
& +\bar{x}^{T}(k)\left(D \otimes A^{T} P B F+D \widetilde{L} \otimes K^{T} B^{T} P B F\right) e(k) \\
& +e^{T}(k)\left(D \otimes F^{T} B^{T} P A+\widetilde{L} D \otimes F^{T} B^{T} P B K\right) \bar{x}(k) \\
& +e^{T}(k)\left(I_{N} \otimes \alpha(S+H B F)^{T} Q_{1}(S+H B F)-\alpha Q_{1}+F^{T} B^{T} P B F\right) e(k) \\
= & \bar{\eta}^{T}(k)\left(\begin{array}{cc}
\Xi_{1} & * \\
*_{1}^{T} & \Xi_{2}
\end{array}\right) \bar{\eta}(k),
\end{aligned}
$$

where

$$
\begin{aligned}
& \Xi_{1}=I_{N} \otimes A^{T} P A+2 D \widetilde{L} D \otimes A^{T} P B K+(D L D)^{2} \otimes K^{T} B^{T} P B K-I_{N} \otimes P, \\
& \Xi_{2}=I_{N} \otimes\left(\alpha(S+H B F)^{T} Q_{1}(S+H B F)-\alpha Q_{1}+F^{T} B^{T} P B F\right), \\
& *_{1}=D \otimes A^{T} P B F+D \widetilde{L} \otimes K^{T} B^{T} P B F .
\end{aligned}
$$

Since $D \widetilde{L} D$ is a symmetric matrix, there exists an orthodox matrix $\Upsilon$ such that $\Upsilon^{T}(D \widetilde{L} D) \Upsilon=\Lambda$, where $\Lambda=\operatorname{diag}\left(\lambda_{1}(D \widetilde{L} D), \lambda_{2}(D \widetilde{L} D), \ldots, \lambda_{N}(D \widetilde{L} D)\right)$. Moreover, by Assumption 1, (i), and Lemma 1, one has a sequence of eigenvalues $\quad 0<\lambda_{1}(D \widetilde{L} D) \leq \lambda_{2}(D \widetilde{L} D) \leq \ldots \leq \lambda_{N}(D \widetilde{L} D)$. Then, let $\check{x}(k)=\left(\Upsilon^{T} \otimes I_{n}\right) \bar{x}(k)$; by (iii) and Lemma 2 , one has

$$
\begin{aligned}
\bar{x}^{T}(k) \Xi_{1} \bar{x}(k) & =\hat{x}^{T}(k)\left(I_{N} \otimes A^{T} P A+2 \Lambda \otimes A^{T} P B K+\Lambda^{2} \otimes K^{T} B^{T} P B K-I_{N} \otimes P\right) \hat{x}(k) \\
& \leq-\check{x}^{T}(k) \check{x}(k)=-\bar{x}^{T}(k) \bar{x}(k) .
\end{aligned}
$$


Meanwhile, by (ii), there exists a positive definite matrix $Q_{1}>0$ such that the following discrete Lyapunov matrix equation holds:

$$
(S+H B F)^{T} Q_{1}(S+H B F)-Q_{1}+I_{l}=0 .
$$

According to (17) and (18), one has

$$
\begin{aligned}
& V(k+1)-V(k) \\
& \quad \leq \bar{\eta}^{T}(k)\left(\begin{array}{cc}
-I_{N n} & *_{1} \\
*_{1}^{T} & -\Psi_{1}
\end{array}\right) \bar{\eta}(k) \\
& =\eta^{T}(k)\left(\begin{array}{cc}
-I_{N n} & *_{2} \\
*_{2}^{T} & -\Psi_{1}
\end{array}\right) \eta(k) \\
& =\eta T(k) \Phi_{1} \eta(k),
\end{aligned}
$$

where $\Phi_{1}=\left(\begin{array}{cc}-I_{N n} & *_{2} \\ *_{2}^{T} & -\Psi_{1}\end{array}\right), \quad \Psi_{1}=I_{N} \otimes\left(\alpha I_{l}-F^{T} B^{T} P B F\right)$ and $*_{2}=\left(D \otimes I_{n}\right) *_{1}$. By Lemma 3 , one has $\Phi_{1}<0$ by choosing sufficiently large constant $\alpha$. Then,

$$
\begin{aligned}
V & (k+1)-V(k) \\
& \leq \lambda_{\max }\left(\Phi_{1}\right) \eta^{T}(k) \eta(k) \\
& =\lambda_{\max }\left(\Phi_{1}\right) \sum_{i=1}^{N}\left(\tilde{x}_{i}^{T}(k) \tilde{x}_{i}(k)+e_{i}^{T}(k) e_{i}(k)\right)
\end{aligned}
$$

$\leq 0$,

where $\quad V(k+1)-V(k)=0$ if and only if $\widetilde{x}_{1}=\widetilde{x}_{2}=\cdots=\widetilde{x}_{N}=0$ and $e_{1}=e_{2}=\cdots=e_{N}=0$. Thus, $\lim _{k \longrightarrow \infty} \tilde{x}_{i}(k)=0, \lim _{k \longrightarrow \infty} e_{i}(k)=0, i=1,2, \ldots, N$, which implies that Theorem 1 holds.

\section{DO-Based Output Feedback Bipartite Consensus}

In this section, a DO-based output feedback protocol is proposed for disturbed linear MASs. State observer and disturbance observers are given, respectively, and the conditions are obtained.

The state observer of the $i$ th follower is designed as

$$
\begin{aligned}
\hat{x}_{i}(k+1)= & A \hat{x}_{i}(k)+B\left(u_{i}(k)+\gamma_{i}(k)\right) \\
& +E\left(C \hat{x}_{i}(k)-y_{i}(k)\right), \quad i=1,2, \ldots, N,
\end{aligned}
$$

and the state observer of the leader is designed as

$$
\hat{x}_{0}(k+1)=A \hat{x}_{0}(k)+E\left(C \hat{x}_{0}(k)-y_{0}(k)\right),
$$

where $\hat{x}_{i}(k)$ is the observed state of the $i$ th follower, $\hat{x}_{0}(k)$ is the observed state of the leader, and $E$ is the gain matrix to be determined.

The discrete-time disturbance observer based on output information is proposed as

$$
\begin{aligned}
v_{i}(k+1)= & (S+H B F)\left(v_{i}(k)-H \hat{x}_{i}(k)\right) \\
& +H\left(A \hat{x}_{i}(k)+B u_{i}(k)+E\left(C \hat{x}_{i}(k)-y_{i}(k)\right)\right), \\
\widehat{\omega}_{i}(k)= & v_{i}(k)-H \hat{x}_{i}(k), \\
\widehat{d}_{i}(k)= & F \widehat{\omega}_{i}(k),
\end{aligned}
$$

where $v_{i} \in R$ is the internal state variable of the observer, $\widehat{\omega}_{i}$ and $\hat{\gamma}_{i}$ are the estimated values of $\omega_{i}$ and $\gamma_{i}$, respectively, and $H \in R^{l \times n}$ is the gain matrix of the observer.

Remark 3. For the case that the state of each agent cannot be obtained, the state observer can be used for estimating the state. Moreover, the disturbances exist in the subsystems. One has to design corresponding controller to attenuate the disturbances. Discrete output-based disturbance observer (23) is proposed.

When the state cannot be obtained, state observer can be used for estimating the state. Therefore, the bipartite consensus protocol can be designed as

$$
u_{i}(k)=K\left[\sum_{j \in N_{i}}\left|a_{i j}\right|\left(\hat{x}_{i}(k)-\operatorname{sgn}\left(a_{i j}\right) \hat{x}_{j}(k)\right)+h_{i}\left(\hat{x}_{i}(k)-d_{i} \hat{x}_{0}(k)\right)\right]-\hat{\gamma}_{i}(k),
$$

where $K$ is the gain to be designed.

Substituting (24) into (1), one has

$$
\begin{aligned}
x_{i}(k+1)= & A x_{i}(k)+B F\left(\omega_{i}(k)-\widehat{\omega}_{i}(k)\right) \\
& +B K\left[\sum_{j \in N_{i}}\left|a_{i j}\right|\left(\hat{x}_{i}(k)-\operatorname{sgn}\left(a_{i j}\right) \hat{x}_{j}(k)\right)+h_{i}\left(\hat{x}_{i}(k)-d_{i} \hat{x}_{0}(k)\right)\right],
\end{aligned}
$$


and then, we give the following result.

Theorem 2. Suppose Assumptions 1-3 hold. The bipartite consensus of MAS (1) with leader (2) will be achieved with state observers (21) and (22) and disturbance observer (23) under bipartite consensus protocol (24) if

(i) Suppose there exists at least one follower pinned by the leader.

(ii) $S+H B F$ is Schur stable.

(iii) $K=-\left(1 / \lambda_{1}(D \tilde{L} D)\right)\left(B^{T} P B+I\right)^{-1} B^{T} P A$, where $P>0$ is the unique solution of algebraic Riccati equation (6), $\lambda_{1}(D \widetilde{L} D)$ is the minimum eigenvalue of $D \widetilde{L} D$, and
Proof. Let $\tilde{x}_{i}(k)=x_{i}(k)-d_{i} x_{0}(k), \tilde{\hat{x}}_{i}(k)=\hat{x}_{i}(k)-d_{i} \hat{x}_{0}(k)$, $\delta_{i}(k)=\tilde{x}_{i}(k)-\hat{x}_{i}(k)$, and $e_{i}(k)=\omega_{i}(k)-\widehat{\omega}_{i}(k)$. By $d_{i} a_{i j} d_{j}=a_{i j} \operatorname{sgn}\left(a_{i j}\right)=\left|a_{i j}\right|$, one has

$$
\begin{aligned}
& \left|a_{i j}\right|\left(\hat{x}_{i}(k)-\operatorname{sgn}\left(a_{i j}\right) \hat{x}_{j}(k)\right) \\
& \quad=\left|a_{i j}\right|\left(\tilde{\hat{x}}_{i}(k)+d_{i} \hat{x}_{0}(k)-\operatorname{sgn}\left(a_{i j}\right) \hat{x}_{j}(k)\right) \\
& \quad=\left|a_{i j}\right|\left(\tilde{\hat{x}}_{i}(k)-\operatorname{sgn}\left(a_{i j}\right) \tilde{\hat{x}}_{j}(k)\right) \\
& \quad=\left|a_{i j}\right|\left(\tilde{x}_{i}(k)-\operatorname{sgn}\left(a_{i j}\right) \tilde{x}_{j}(k)\right) \\
& \quad\left(+\operatorname{sgn}\left(a_{i j}\right) \delta_{j}(k)-\delta_{j}(k)\right),
\end{aligned}
$$

and then, the error system can be written as follows: $D \widetilde{L} D=D L D+\bar{H}, \bar{H}=\operatorname{diag}\left(h_{1}, h_{2}, \ldots, h_{N}\right)$.

$$
\begin{aligned}
\tilde{x}_{i}(k+1)= & A \tilde{x}_{i}(k)+B F e_{i}(k)+B K\left[\sum_{j \in N_{i}}\left|a_{i j}\right|\left(x_{i}(k)-\operatorname{sgn}\left(a_{i j}\right) \tilde{x}_{j}(k)+\operatorname{sgn}\left(a_{i j}\right) \delta_{j}(k)-\delta_{j}(k)\right)\right. \\
& \left.+h_{i}\left(\tilde{x}_{i}(k)-\delta_{i}(k)\right)\right], \\
\tilde{\hat{x}}_{i}(k+1)= & A \tilde{\hat{x}}_{i}(k)+B F\left(e_{i}(k)-E C \delta_{i}(k)\right) \\
& +B K\left[\sum_{j \in N_{i}}\left|a_{i j}\right|\left(\tilde{x}_{i}(k)-\operatorname{sgn}\left(a_{i j}\right) \tilde{x}_{j}(k)+\operatorname{sgn}\left(a_{i j}\right) \delta_{j}(k)-\delta_{j}(k)\right)\right. \\
& \left.+h_{i}\left(\tilde{x}_{i}(k)-\delta_{i}(k)\right)\right], \\
\delta_{i}(k+1)= & (A+E C) \delta_{i}(k), \\
e_{i}(k+1)= & (S+H B F) e_{i}(k) .
\end{aligned}
$$

Denote $\quad \tilde{x}(k)=\left(\tilde{x}_{1}^{T}(k), \tilde{x}_{2}^{T}(k), \ldots, \tilde{x}_{N}^{T}(k)\right)^{T}$, $\delta(k)=\left(\delta_{1}^{T}(k), \delta_{2}^{T}(k), \ldots, \delta_{N}^{T}\right)^{T}$,

$e(k)=\left(e_{1}^{T}(k), e_{2}^{T}(k), \ldots, e_{N}^{T}(k)\right)^{T}$, and (27) can be rewritten as follows:

$$
\begin{aligned}
\tilde{x}(k+1)= & \left(I_{N} \otimes A+L \otimes B K\right) \tilde{x}(k)+\left(I_{N} \otimes B F\right) e(k) \\
& -(L \otimes B K) \delta(k), \\
\delta(k+1)= & \left(I_{N} \otimes(A+E C)\right) \delta(k), \\
e(k+1)= & \left(I_{N} \otimes(S+H B F)\right) e(k) .
\end{aligned}
$$

Consider the following Lyapunov candidate function:

$$
\begin{aligned}
V(k)= & \zeta^{T}(k)\left(\begin{array}{ccc}
I_{N} \otimes P & 0 & 0 \\
0 & \beta I_{N} \otimes Q_{2} & 0 \\
0 & 0 & \beta I_{N} \otimes Q_{3}
\end{array}\right) \zeta(k) \\
= & \tilde{x}^{T}(k)\left(I_{N} \otimes P\right) \widetilde{x}(k)+\beta \delta^{T}(k)\left(I_{N} \otimes Q_{2}\right) \delta(k) \\
& +\beta e^{T}(k)\left(I_{N} \otimes Q_{3}\right) e(k),
\end{aligned}
$$

where $\zeta(k)=\left(\tilde{x}^{T}(k), \delta^{T}(k), e^{T}(k)\right)^{T}, Q_{2}$ and $Q_{3}$ are positive definite matrices which were designed later, and $\beta>0$ is a sufficiently large constant.

Let $\bar{x}(k)=\left(D \otimes I_{n}\right) \tilde{x}(k), \bar{\zeta}(k)=\left(\bar{x}^{T}(k), \delta^{T}(k), e^{T}(k)\right)^{T}$, and then 


$$
\begin{aligned}
V & (k+1)-V(k) \\
& =\bar{x}^{T}(k)\left(I_{N} \otimes A^{T} P A+2 D \widetilde{L} D \otimes A^{T} P B K\right. \\
& \left.+(D L D)^{2} \otimes K^{T} B^{T} P B K-I_{N} \otimes P\right) \bar{x}(k) \\
& -\bar{x}^{T}(k)\left(D \widetilde{L} \otimes A^{T} P B K+D \widetilde{L}^{2} \otimes K^{T} B^{T} P B K\right) \delta(k) \\
& -\delta^{T}(k)\left(\widetilde{L} D \otimes K^{T} B^{T} P A+\widetilde{L}^{2} D \otimes K^{T} B^{T} P B K\right) \bar{x}(k) \\
& +\bar{x}^{T}(k)\left(D \otimes A^{T} P B F+D \widetilde{L} \otimes K^{T} B^{T} P B F\right) e(k) \\
& +e^{T}(k)\left(D \otimes F^{T} B^{T} P A+\widetilde{L} D \otimes F^{T} B^{T} P B K\right) \bar{x}(k) \\
& -\delta^{T}(k)\left(\widetilde{L} \otimes K^{T} B^{T} P B F\right) e(k)-e^{T}(k)\left(\widetilde{L} \otimes F^{T} B^{T} P \times B K\right) \\
& +\delta(k)+\delta^{T}(k)\left(\beta I _ { N } \otimes \left((A+E C)^{T} Q_{2}(A+E C)\right.\right. \\
& \left.\left.-Q_{2}\right)+\widetilde{L}^{2} \otimes K^{T} B^{T} P B K\right) \delta(k)+e^{T}(k)\left(I_{N} \otimes(\beta(S\right. \\
& \left.\left.+H B F)^{T} Q_{3}(S+H B F)-\beta Q_{3}+F^{T} B^{T} P B F\right)\right) e(k) \\
& =\bar{\zeta}^{T}(k)\left(\begin{array}{ccc}
\Xi_{3} & *_{3} & *_{4} \\
*_{3}^{T} & \Xi_{4} & *_{5} \\
*_{4}^{T} & *_{5}^{T} & \Xi_{5}
\end{array}\right) \bar{\zeta}(k),
\end{aligned}
$$

where

$$
\begin{aligned}
\Xi_{3}= & I_{N} \otimes A^{T} P A+2 D \widetilde{L} D \otimes A^{T} P B K \\
& +(D L D)^{2} \otimes K^{T} B^{T} P B K-I_{N} \otimes P, \\
\Xi_{4}= & \beta I_{N} \otimes\left((A+E C)^{T} Q_{2}(A+E C)-Q_{2}\right)+\widetilde{L}^{2} \otimes K^{T} B^{T} P B K, \\
\Xi_{5}= & I_{N} \otimes\left(\beta(S+H B F)^{T} Q_{3}(S+H B F)-\beta Q_{3}+F^{T} B^{T} P B F\right), \\
*_{3}= & D \widetilde{L} \otimes A^{T} P B K+D \widetilde{L}^{2} \otimes K^{T} B^{T} P B K, \\
*_{4}= & D \otimes A^{T} P B F+D \widetilde{L} \otimes K^{T} B^{T} P B F, \\
*_{5}= & \widetilde{L} \otimes K^{T} B^{T} P B F .
\end{aligned}
$$

And, under Assumption 3, the gain matrix $E$ can be selected such that $A+E C$ is Schur.

Similar to (17), there exists an orthodox matrix $\Upsilon$ such that

$$
\Upsilon^{T}(D \widetilde{L} D) \Upsilon=\Lambda,
$$

where

$\Lambda=\operatorname{diag}\left(\lambda_{1}(D \widetilde{L} D), \lambda_{2}(D \widetilde{L} D), \ldots, \lambda_{N}(D \widetilde{L} D)\right)$.

Let

$\check{x}(k)=\left(\Upsilon^{T} \otimes I_{n}\right) \bar{x}(k)$. By (iii) and Lemma 2 , one has

$$
\bar{x}^{T}(k) \Xi_{3} \bar{x}(k) \leq-\check{x}^{T}(k) \check{x}(k)=-\bar{x}^{T}(k) \bar{x}(k),
$$

and since matrices $A+E C$ and $S+H B F$ are Schur, there exist, respectively, positive definite matrices $Q_{2}$ and $Q_{3}$ such that the discrete Lyapunov matrix equations hold as follows:

$$
\begin{gathered}
(A+E C)^{T} Q_{2}(A+E C)-Q_{2}+I_{n}=0, \\
(S+H B F)^{T} Q_{3}(S+H B F)-Q_{3}+I_{l}=0 .
\end{gathered}
$$

According to (32)-(34), one has

$$
\begin{aligned}
& V(k+1)-V(k) \\
& \leq \bar{\zeta}^{T}(k)\left(\begin{array}{ccc}
-I_{N n} & *_{3} & *_{4} \\
*_{3}^{T} & -\Psi_{2} & *_{5} \\
*_{4}^{T} & *_{5}^{T} & -\Psi_{3}
\end{array}\right) \bar{\zeta}(k) \\
& \quad=\zeta^{T}(k)\left(\begin{array}{ccc}
-I_{N n} & *_{6} & *_{7} \\
*_{6}^{T} & -\Psi_{2} & *_{5} \\
*_{7}^{T} & *_{5}^{T} & -\Psi_{3}
\end{array}\right) \zeta(k) \\
& =\zeta^{T}(k) \Phi_{2} \zeta(k),
\end{aligned}
$$

where

$$
\begin{aligned}
& \Phi_{2}=\left(\begin{array}{ccc}
-I_{N n} & *_{6} & *_{7} \\
*_{6}^{T} & -\Psi_{2} & *_{5} \\
*_{7}^{T} & *_{5}^{T} & -\Psi_{3}
\end{array}\right), \\
& \Psi_{2}=\beta I_{N n}-\widetilde{L}^{2} \otimes K^{T} B^{T} P B K, \\
& \Psi_{3}=I_{N} \otimes\left(\beta I_{l}-F^{T} B^{T} P B F\right), \\
& *_{6}=\left(D \otimes I_{n}\right) *_{3}, \\
& *_{7}=\left(D \otimes I_{n}\right) *_{4} .
\end{aligned}
$$

By choosing sufficiently large constant $\beta$, according to Lemma 3, one has $\Phi_{2}<0$. Then,

$$
\begin{aligned}
& V(k+1)-V(k) \\
& \quad \leq \lambda_{\max }\left(\Phi_{2}\right) \zeta^{T}(k) \zeta(k) \\
& \quad=\lambda_{\max }\left(\Phi_{2}\right) \sum_{i=1}^{N}\left(\widetilde{x}_{i}^{T}(k) \widetilde{x}_{i}(k)+\delta_{i}^{T}(k) \delta_{i}(k)+e_{i}^{T}(k) e_{i}(k)\right) \\
& \quad \leq 0,
\end{aligned}
$$

where $\quad V(k+1)-V(k)=0$ if and only if $\tilde{x}_{1}=\tilde{x}_{2}=\cdots=\tilde{x}_{N}=0, \quad \delta_{1}(k)=\delta_{2}(k)=\cdots=\delta_{N}(k)=0$, and $e_{1}=e_{2}=\cdots=e_{N}=0$. Thus, $\quad \lim _{k \rightarrow \infty} \widetilde{x}_{i}(k)=0$, $\lim _{k \longrightarrow \infty} \delta_{i}(k)=0, \quad \lim _{k \longrightarrow \infty} e_{i}(k)=0 . \quad$ Moreover, $\lim _{k \rightarrow \infty} \hat{\hat{x}}_{i}(k)=0, \quad i=1,2, \ldots, N$. Thus, Theorem 2 holds.

\section{Simulations}

In this section, we give two simulation examples to illustrate the theoretical results of Sections 3 and 4. In Figure 1, consider seven agents composed of six followers with one leader in a network with competitive interaction. Moreover, we choose $n=2$ and $m=3$ as the dimensions of the state and output of the seven agents, respectively.

Example 1. (the case of state feedback) In Figure 1, note that the signed topology is connected and the bipartition $\mathscr{V}_{1}=\{1,2,3\}, \mathscr{V}_{2}=\{4,5,6\}$ satisfies Definition 1. Therefore, Assumption 1 is satisfied. Moreover, the matrix $D$ can be selected as $D=\operatorname{diag}(1,1,1,-1,-1,-1)$. Meanwhile, the matrices $\widetilde{L}$ and $D \widetilde{L} D$ can be calculated as follows: 


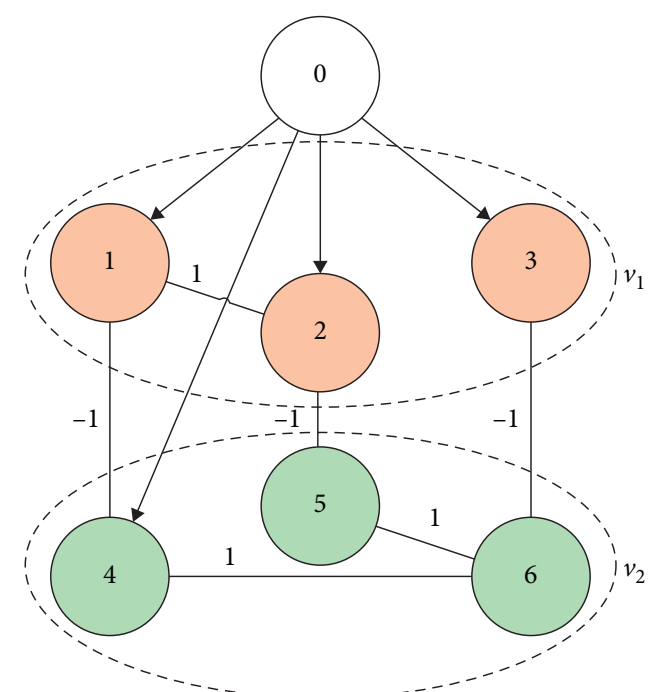

FIGURE 1: A network with competitive interactions containing 7 agents.

$$
\begin{aligned}
\widetilde{L} & =\left(\begin{array}{cccccc}
3 & -1 & 0 & 1 & 0 & 0 \\
-1 & 3 & 0 & 0 & 1 & 0 \\
0 & 0 & 2 & 0 & 0 & 1 \\
1 & 0 & 0 & 3 & 0 & -1 \\
0 & 1 & 0 & 0 & 2 & -1 \\
0 & 0 & 1 & -1 & -1 & 3
\end{array}\right), \\
D \widetilde{L} D & =\left(\begin{array}{cccccc}
3 & -1 & 0 & -1 & 0 & 0 \\
-1 & 3 & 0 & 0 & -1 & 0 \\
0 & 0 & 2 & 0 & 0 & -1 \\
-1 & 0 & 0 & 3 & 0 & -1 \\
0 & -1 & 0 & 0 & 2 & -1 \\
0 & 0 & -1 & -1 & -1 & 3
\end{array}\right),
\end{aligned}
$$

and one can obtain $\lambda_{1}(D \widetilde{L} D)=0.5395$.

Then, choose the following system matrices:

$$
\begin{aligned}
& A=\left(\begin{array}{cc}
0.9 & -2 \\
0 & -1.01
\end{array}\right), \\
& B=\left(\begin{array}{c}
0.02 \\
-0.05
\end{array}\right) \\
& F=\left(\begin{array}{ll}
-0.03 & 1.8
\end{array}\right), \\
& S=\left(\begin{array}{cc}
-1 & 0 \\
0 & 0
\end{array}\right)
\end{aligned}
$$

where matrices $A$ and $B$ satisfy Assumption 2. And the gain matrix $H$ can be selected as follows:

$$
H=\left(\begin{array}{cc}
-0.2 & 0 \\
0 & -0.5
\end{array}\right),
$$

and thus one has that the matrix $S+H B F$ is Schur. By algebraic Riccati equation (6), one has

$$
P=\left(\begin{array}{cc}
4.8332 & -4.7634 \\
-4.7634 & 40.0959
\end{array}\right) \text {, }
$$

and thus the gain matrix $K$ of bipartite consensus protocol (10) can be obtained:

$$
K=\left(\begin{array}{ll}
-0.5025 & -2.4200
\end{array}\right) .
$$

For the MAS (1) with leader (2) and exogenous system (3), the initial values of $x_{i}(k), x_{0}(k)$, and $\omega_{i}(k)$ are given as follows:

$$
\begin{aligned}
& x_{1}(1)=(9,10)^{T}, \\
& x_{2}(1)=(-16,-4)^{T}, \\
& x_{3}(1)=(12,-15)^{T}, \\
& x_{4}(1)=(-11,-9)^{T}, \\
& x_{5}(1)=(-9,14)^{T}, \\
& x_{6}(1)=(13,7)^{T}, \\
& x_{0}(1)=(1.5,-1.5)^{T}, \\
& \omega_{1}(1)=(2,2)^{T}, \\
& \omega_{2}(1)=(-1,-1.2)^{T}, \\
& \omega_{3}(1)=(-3,-3)^{T}, \\
& \omega_{4}(1)=(5,4)^{T}, \\
& \omega_{5}(1)=(-2,-2)^{T}, \\
& \omega_{6}(1)=(1,1)^{T},
\end{aligned}
$$

where $i=1,2, \ldots, 6$. In Figures 2 and 3, the trajectories of $x_{i}(k), i=1,2, \ldots, 6$, are displayed. And one can obtain that the bipartite consensus of MAS (1) with leader (2) can be achieved under bipartite consensus protocol (10) by Figures 2 and 3. Thus, the effectiveness of Theorem 1 is verified.

Example 2. (the case of output feedback) In this case, consider the same system matrices $A, B, F, S$ and the gain matrix $H$ as Example 1, and one has that Assumption 1 can be satisfied and the solution $P>0$ of algebraic Riccati equation (6) and the same gain matrix $K$ as Example 1 can be calculated. Moreover, we can obtain that the matrix $S+H B F$ is Schur. Then, choose the output matrix $C$ as follows:

$$
C=\left(\begin{array}{cc}
-0.5 & 0 \\
0 & 0.5 \\
0 & 0
\end{array}\right),
$$

and thus the matrix pair $(A, C)$ is detectable satisfying Assumption 3. Meanwhile, the matrix $E$ can be selected as follows:

$$
E=\left(\begin{array}{lll}
1 & 0 & 0 \\
0 & 1 & 0
\end{array}\right),
$$

and then one has that $A+E C$ is Schur. 


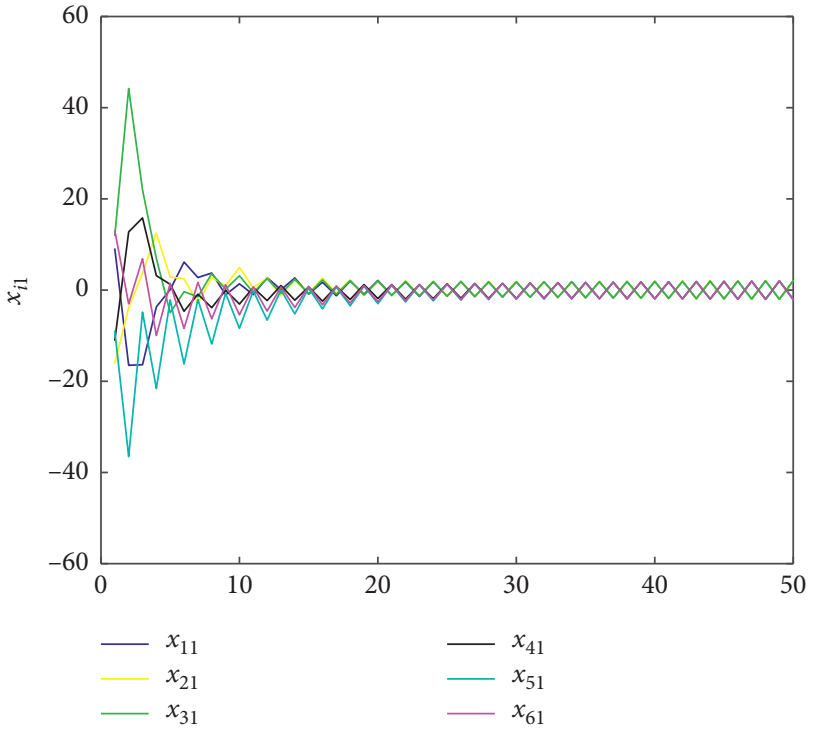

FigURE 2: The trajectories of $x_{i 1}, i=1,2, \ldots, 6$, under state feedback protocol.

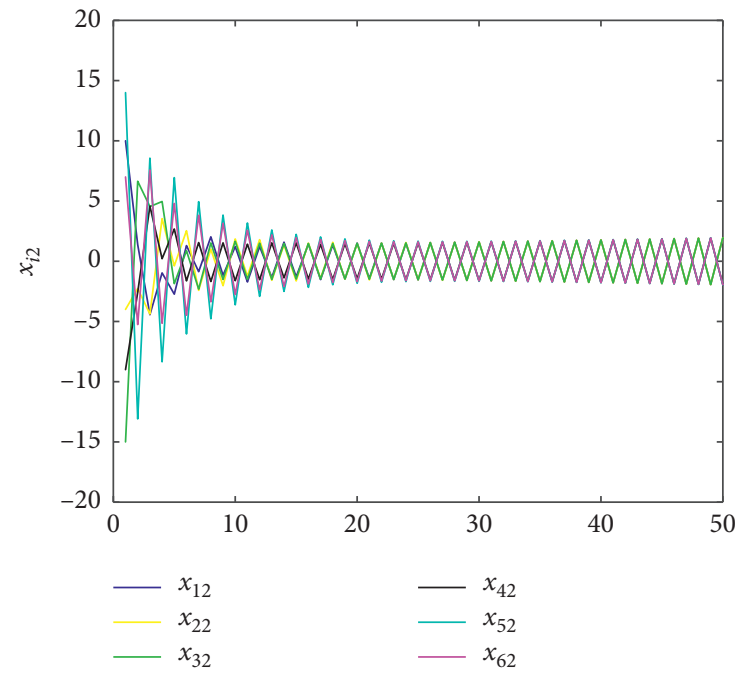

FigURE 3: The trajectories of $x_{i 2}, i=1,2, \ldots, 6$, under state feedback protocol.

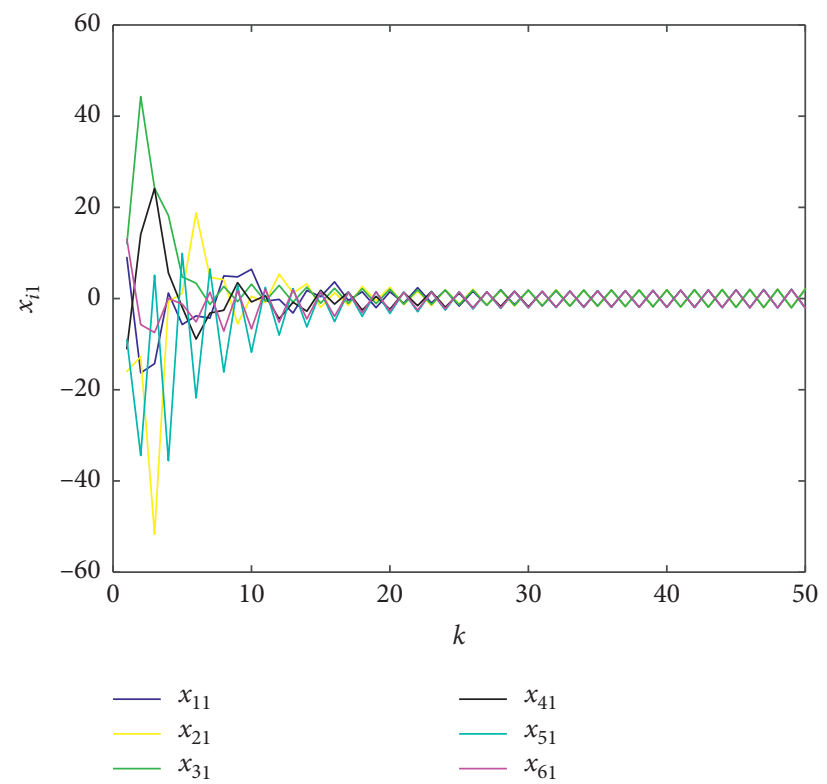

Figure 4: The trajectories of $x_{i 1}, i=1,2, \ldots, 6$, under output feedback protocol. 


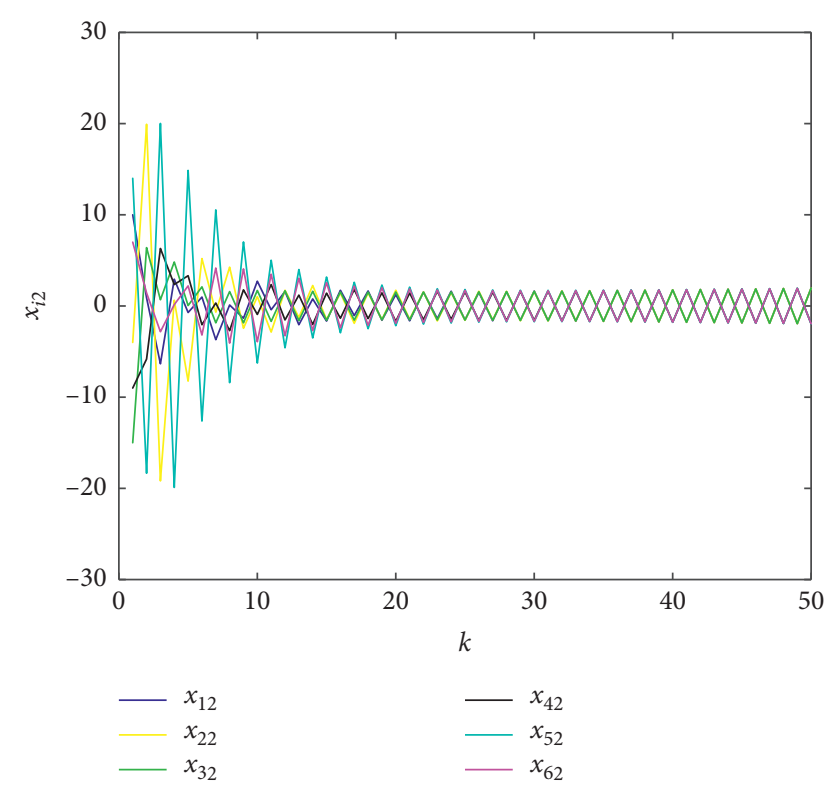

Figure 5: The trajectories of $x_{i 2}, i=1,2, \ldots, 6$, under output feedback protocol.

For MAS (1) with leader (2) and exogenous system (3), we choose the same initial values of $x_{i}(k), x_{0}(k)$, and $\omega_{i}(k)$ as Example 1 and the observed values of $\hat{x}_{i}(k), \hat{x}_{0}(k)$ as follows: $\hat{x}_{1}(1)=(14,13)^{T}, \hat{x}_{2}(1)=(18,17)^{T}, \hat{x}_{3}(1)=(-$ $10,-11)^{T}, \quad \hat{x}_{4}(1)=(-14,-12)^{T}, \quad \hat{x}_{5}(1)=(-16,-17)^{T}$, $\hat{x}_{6}(1)=(11,14)^{T}, \hat{x}_{0}(1)=(-0.1,-0.1)^{T}$. In Figures 4 and 5 , the trajectories of $x_{i}(k)$ are presented, and one can note that the bipartite consensus can be achieved for MAS (1) with leader (2) via bipartite consensus protocol (24). Thus, the effectiveness of Theorem 2 is verified.

\section{Conclusions}

In this paper, bipartite consensus is investigated for discretetime MASs with exogenous disturbances. With the help of DTDO proposed in this paper, both the state feedback and the output feedback protocols are given, in which the gains can be determined by solving some discrete-time algebraic Riccati equations. Then, using stability theory, some sufficient conditions are obtained. Finally, numerical simulations are presented to illustrate the theoretical findings.

\section{Data Availability}

No data were used to support this study.

\section{Conflicts of Interest}

The authors declare that there are no conflicts of interest.

\section{Acknowledgments}

This study was partially supported by the Natural Science Foundation of Hunan Province (2020JJ6089) and the Key Project of the Department of Education in Hunan Province (19A133).

\section{References}

[1] H. Zhang, X. Zhou, Z. Wang, H. Yan, and J. Sun, "Adaptive consensus-based distributed target tracking with dynamic cluster in sensor networks," IEEE Transactions on Cybernetics, vol. 49, no. 5, pp. 1580-1591, 2019.

[2] D. Wang, Z. Wang, C. Wen, and W. Wang, "Second-order continuous-time algorithm for optimal resource allocation in power systems," IEEE Transactions on Industrial Informatics, vol. 15, no. 2, pp. 626-637, 2019.

[3] H. Yan, X. Zhou, H. Zhang, F. Yang, and Z.-G. Wu, "A novel sliding mode estimation for microgrid control with communication time delays," IEEE Transactions on Smart Grid, vol. 10, no. 2, pp. 1509-1520, 2019.

[4] D. Ding, Z. Wang, and Q.-L. Han, “A set-membership approach to event-triggered filtering for general nonlinear systems over sensor networks," IEEE Transactions on Automatic Control, vol. 65, no. 4, pp. 1792-1799, 2020.

[5] A. Jadbabaie, J. Jie Lin, and A. S. Morse, "Coordination of groups of mobile autonomous agents using nearest neighbor rules," IEEE Transactions on Automatic Control, vol. 48, no. 6, pp. 988-1001, 2003.

[6] W. Ren and R. Beard, "Consensus seeking in multi-agent systems under dynamically changing interaction topologies," IEEE Transactions on Automatic Control, vol. 50, no. 5, pp. 655-661, 2005.

[7] Z. Li and J. Chen, "Robust consensus of linear feedback protocols over uncertain network graphs," IEEE Transactions on Automatic Control, vol. 62, no. 8, pp. 4251-4258, 2017.

[8] H. Su, H. Wu, and J. Lam, "Positive edge-consensus for nodal networks via output feedback," IEEE Transactions on Automatic Control, vol. 64, no. 3, pp. 1244-1249, 2019.

[9] X. Wang, G.-P. Jiang, H. Su, and Z. Zeng, "Consensus-based distributed reduced-order observer design for LTI systems," IEEE Transactions on Cybernetics, pp. 1-11, 2020.

[10] X. Wang, X. Wang, H. Su, and J. Lam, "Coordination control for uncertain networked systems using interval observers," IEEE Transactions on Cybernetics, vol. 50, no. 9, pp. 40084019, 2020.

[11] C. Xu, Y. Zhao, B. Qin, and H. Zhang, "Adaptive synchronization of coupled harmonic oscillators under switching topology," Journal of the Franklin Institute, vol. 356, no. 2, pp. 1067-1087, 2019.

[12] Y. Liu and H. Su, "Some necessary and sufficient conditions for containment of second-order multi-agent systems with sampled position data," Neurocomputing, vol. 378, pp. 228237, 2020.

[13] Y. Liu and H. Su, "Containment control of second-order multi-agent systems via intermittent sampled position data communication," Applied Mathematics and Computation, vol. 362, Article ID 124522, 2019.

[14] B. Cheng and Z. Li, "Fully distributed event-triggered protocols for linear multiagent networks," IEEE Transactions on Automatic Control, vol. 64, no. 4, pp. 1655-1662, 2019.

[15] S. Chen, H. Pei, Q. Lai, and H. Yan, "Multitarget tracking control for coupled heterogeneous inertial agents systems based on flocking behavior," IEEE Transactions on Systems, Man, and Cybernetics: Systems, vol. 49, no. 12, pp. 2605-2611, 2019.

[16] X. Wang and H. Su, "Completely model-free RL-based consensus of continuous-time multi-agent systems," Applied Mathematics and Computation, vol. 382, Article ID 125312, 2020. 
[17] C. Altafini, "Consensus problems on networks with antagonistic interactions," IEEE Transactions on Automatic Control, vol. 58, no. 4, pp. 935-946, 2013.

[18] L. Tian, Z. Ji, T. Hou, and K. Liu, "Bipartite consensus on coopetition networks with time-varying delays," IEEE Access, vol. 6, no. 1, pp. 10169-10178, 2018.

[19] L. Tian, Z. J. Ji, T. Hou, and H. Xu, "Bipartite consensus of edge dynamics on coopetition multi-agent systems," Science China: Information Sciences, vol. 62, no. 12, Article ID 229201, 2019.

[20] J. Qin, W. Fu, W. Zheng, and H. Gao, "On the bipartite consensus for generic linear multiagent systems with input saturation," IEEE Transactions on Cybernetics, vol. 47, no. 8, pp. 1948-1958, 2016.

[21] J. Hu, Y. Wu, T. Li, and B. K. Ghosh, "Consensus control of general linear multiagent systems with antagonistic interactions and communication noises," IEEE Transactions on Automatic Control, vol. 64, no. 5, pp. 2122-2127, 2019.

[22] J. Li, X. Chen, F. Hao, and J. Xie, "Event-triggered bipartite consensus for multi-agent systems with antagonistic interactions," International Journal of Control, Automation and Systems, vol. 17, pp. 1-13, 2019.

[23] H. Zhang, Y. Cai, Y. Wang, and H. Su, "Adaptive bipartite event-triggered output consensus of heterogeneous linear multiagent systems under fixed and switching topologies," IEEE Transactions on Neural Networks and Learning Systems, vol. 31, no. 11, pp. 4816-4830, 2020.

[24] D. Meng, Y. Jia, and J. Du, "Finite-time consensus for multiagent systems with cooperative and antagonistic interactions," IEEE Transactions on Neural Networks and Learning Systems, vol. 27, no. 4, pp. 762-770, 2016.

[25] X. Wang, G.-P. Jiang, H. Su, and X. Wang, "Robust global coordination of networked systems with input saturation and external disturbances," IEEE Transactions on Systems, Man, and Cybernetics: Systems, pp. 1-13, 2020.

[26] L. Guo and W.-H. Chen, "Disturbance attenuation and rejection for systems with nonlinearity via DOBC approach," International Journal of Robust and Nonlinear Control, vol. 15, no. 3, pp. 109-125, 2005.

[27] H. Yang, Z. Zhang, and S. Zhang, "Consensus of second-order multi-agent systems with exogenous disturbances," International Journal of Robust and Nonlinear Control, vol. 21, no. 9, pp. 945-956, 2011.

[28] C. Xu, Y. Zheng, H. Su, and H.-B. Zeng, "Containment for linear multi-agent systems with exogenous disturbances," Neurocomputing, vol. 160, pp. 206-212, 2015.

[29] C. Xu, H. Xu, H. Su, and C. Liu, "Disturbance-observer based consensus of linear multi-agent systems with exogenous disturbance under intermittent communication," Neurocomputing, vol. 404, pp. 26-33, 2020.

[30] Y. Wang, Z. Wang, and L. Kong, "Robust synchronization of class chaotic systems using novel time-varying gain disturbance observer-based sliding mode control," Complexity, vol. 2021, Article ID 8845553, 14 pages, 2021.

[31] M. Chen, S. Xiong, and Q. Wu, "Tracking flight control of quadrotor based on disturbance observer," IEEE Transactions on Systems, Man, and Cybernetics: Systems, vol. 51, no. 3, pp. 1414-1423, 2021.

[32] H. Xu, C. Liu, Y. Lv, and J. Zhou, "Adaptive bipartite consensus of second-order multi-agent systems with bounded disturbances," IEEE Access, vol. 8, pp. 186441-186447, 2020.

[33] Z. Lin, Low Gain Feedback, Lecture Notes in Control and Information Sciences, Springer, London, UK, 1998.
[34] S. Boyd, L. Ghaoui, E. Feron, and V. Balakrishnan, Linear Matrix Inequalities in System and Control Theory, SIAM, Philadelphia, PA, USA, 1994. 